\title{
Behavior of open sets in bi-Alexandroff topological space
}

\author{
D. Sasikala ${ }^{1 *}$ and A. Divya ${ }^{2}$
}

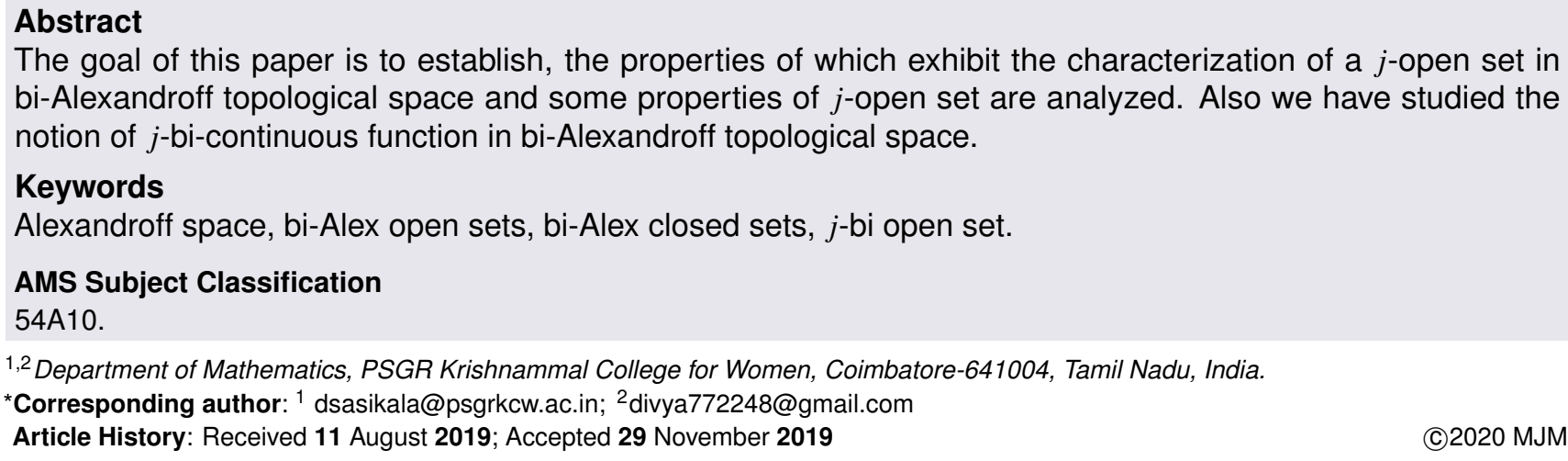

\section{Contents}

1 Introduction 48

2 Preliminaries 48

bi-Alexandroff topological space $\ldots \ldots \ldots \ldots \ldots \ldots 49$

pre-bi open set and pre-bi closed set ............ 50

j-open set on bi-Alexandroff topological space ... 51

$j$-bi-continuous function ..................... 51

Rules for constructing $j$-bi-continuous function .. 52

References

\section{Introduction}

Nowadays the topological structures have many applications in our daily life. Single topology is extended to bitopology, tri topology and quad topology with usual definitions. The concept of bitopological space was first introduced by Kelly [5], and tritopological spaces was implemented by Martin M. Kovar [8]. The quad topological space was invented by Dhanya V. Mukundan [4]. These papers are pushup to study us Alexandroff topological space into bi-Alexandroff topological space.

Alexandroff topological space was first expossed by $\mathrm{P}$. Alexandroff in 1937 [1]. The Alexandroff space (a space with the condition of Alexandroff) is a topological space such that arbitrary intersection of open sets is open (the union of any number of closed sets is closed), equivalently, every point has a minimal neighborhood $V(x)$ and is that the intersection of all open sets containing $x$, or equivalently it has an unique minimal base [2].

The Alexandroff topological space was a consequence of the important role of finite spaces in digital topology and therefore the undeniable fact that these spaces have all properties of finite spaces [7], [10]. D. Sasikala and I. Arockiarani initiated $\lambda_{\alpha}$-j-closed sets in generalized topological spaces in 2011 [11]. Then they also initiated decomposition of $j$-closed sets in bigeneralized topological space in 2012 [12].

Furthermore $j$-sets have motivated us to analyze $j$-sets in bi-Alexandroff topological space. Our purpose of this paper is to develop the basic concepts and properties of bi-Alexandroff topological space by using $j$-sets.

\section{Preliminaries}

Definition 2.1. [1] Let us consider X be a topological space, then $X$ is an Alexandroff space if arbitrary intersection of open sets is open.

Theorem 2.2. [13] Let us consider $X$ be a metric space, then $X$ is an Alexandroff space if and only if $X$ has discrete topology.

Definition 2.3. [5] If $X$ is any set, a basis for the topology on the set $X$ is a collection $\mathscr{B}$ of subsets of $X$ called basis elements such that,

(1) For each $x \in X$, there is atleast one basis element B containing $x$. 
(2) If $x$ belongs to the intersection of two basis element $B_{1}$ and $B_{2}$, then there is a basis element $B_{3}$ containing $x$ such that $B_{3} \subset B_{1} \cap B_{2}$.

Theorem 2.4. [13] If $X$ is an Alexandroff space with topology $\tau$ then,

$$
\beta=\{S(x) \quad \mid x \in X\}
$$

is a basis for $\tau$.

Definition 2.5. [5] Let us consider $X$ be a topological space with $\tau$. If $Y$ is a subset of $X$, the collection

$$
\tau_{Y}=\{Y \cap U \quad \mid \quad U \in \tau\}
$$

is a topology on $Y$, called the subspace topology with this topology, $Y$ is called a subspace of $X$.

Definition 2.6. [5] If $\mathscr{B}$ is basis for the topology on a set $X$ then,

$$
\mathscr{B}_{Y}=\{B \cap Y \quad \mid \quad B \in \mathscr{B}\}
$$

is a basis for the subspace topology on $Y$.

Definition 2.7. [9] A subset $S$ of a space $X$ is said to be a pre-open set if $A \subseteq \operatorname{int}(\operatorname{cl}(S))$.

Definition 2.8. [11] A subset $S$ of a space $X$ is said to be a j-open set if $A \subseteq \operatorname{int}(\operatorname{precl}(S))$.

\section{3. bi-Alexandroff topological space}

Definition 3.1. Let a non-empty set be $X, A_{\tau_{1}}$ and $A_{\tau_{2}}$ are Alexandroff topologies on $X$. Then a subset $S$ of $X$ is said to be a bi-Alex open set (briefly $A_{\tau_{1} \tau_{2}}$-open set) if $S \in A_{\tau_{1}} \cap A_{\tau_{2}}$ and complement is said to be a bi-Alex closed set (briefly $A_{\tau_{1} \tau_{2}}$-closed set).

Obviously by the definition of bi-alex open sets it satisfies all the axioms of Alexandroff topological space, in which Alexandroff topology is denoted by $A_{\tau_{1}} \cap A_{\tau_{2}}$ and $X$ with two topologies called bi-Alexandroff topological space and denoted by $\left(X, A_{\tau_{1}}, A_{\tau_{2}}\right)$.

Example 3.2. Let $X=\{a, b, c, d\}, A_{\tau_{1}}=\{X, \emptyset,\{b\},\{d\}$, $\{a, d\},\{b, d\},\{a, b, d\}\}, A_{\tau_{2}}=\{X, \emptyset,\{b\},\{d\},\{a, d\},\{b, d\}$, $\{a, b, d\},\{c\},\{b, c\},\{c, d\},\{a, c, d\},\{b, c, d\}\}$.

Then bi-Alex open sets are $X, \emptyset,\{b\},\{d\},\{a, d\},\{b, d\},\{a, b, d\}$ bi-Alex closed sets are $X, \emptyset,\{a, c, d\},\{a, b, c\},\{b, c\},\{a, c\},\{c\}$.

Definition 3.3. A subset $S$ of a bi-Alexandroff topological space $\left(X, A_{\tau_{1}}, A_{\tau_{2}}\right)$ is called bi-Alex neighborhood of a point $x \in X$ if and only if there exists a bi-Alex open set $U$ such that $x \subset U \subset S$.

Definition 3.4. Let a bi-Alexandroff topological space be $\left(X, A_{\tau_{1}}, A_{\tau_{2}}\right)$ and take $S \subset X$. Then the intersection of biAlex closed sets containing $S$ is called a bi-Alex closure of $S$ and denoted by bi-cl(S) and the union of bi-Alex open sets contained in $S$ is called a bi-Alex interior of $S$ and denoted by bi-int $(S)$.
Theorem 3.5. $X$ is a bi-Alexandroff topological space if and only iffor any $x \in X$ has a minimal bi-Alex open neighborhood

Proof. Assume that $X$ is a bi-Alexandroff topology with $y \in$ $X$. Let $O(y)=\left\{U \subset X: U\right.$ is an open neighborhood of $y$ in $A_{\tau_{1}}$ and $\left.A_{\tau_{2}}\right\}$. Let $S(y)=\cap U$ for $U \in O(y)$, then $S(y)$ is an open neighborhood of $y$ in $A_{\tau_{1}}$ and $A_{\tau_{2}}$, because $X$ is bi-Alexandroff topology. Since $S(y)=\cap U$ it is clear that $S(y)$ is a minimal bi-Alex open neighborhood of $y$.

Conversely, suppose that for each $y \in X$ has a minimal biAlex open neighborhood $S(y)$, then an arbitrary intersection of bi-Alex open sets $V=\bigcap_{\alpha \in A} U_{\alpha}$, where $U_{\alpha}$ is open in $A_{\tau_{1}}$ and $A_{\tau_{2}}$. If $V$ is empty set then it completes the proof. If not, then pick $y \in V$ and we have $y \in U_{\alpha}, \forall \alpha \in A$. Hence $S(y) \subset U_{\alpha} \forall \alpha$, because $S(y)$ is the minimal bi-Alex open neighborhood. Therefore $S(y) \subset V$. Hence $V$ is open in $A_{\tau_{1}}$ and $A_{\tau_{2}}$. Therefore $V$ is bi-Alex open set, since it contains an bi-Alex open set around each of it's point.

Definition 3.6. Let $\left(X, A_{\tau_{1}}, A_{\tau_{2}}\right)$ be a bi-Alexandroff topological space. If $S$ is a subset of $X$, then the collection

$$
\tau_{S}=\{S \cap G \quad \mid \quad G \text { is bi-Alex open in } X\}
$$

is a Alexandroff topology on $S$ called the subspace bi-Alexandroff topology and with this topology $\tau_{S}, S$ is called a subspace of $X$.

Example 3.7. Let $X=\{1,2,3,4\}, A_{\tau_{1}}=\{X, \emptyset,\{1\},\{2\}$, $\{1,2\},\{1,2,3\}\}, A_{\tau_{2}}=\{X, \emptyset,\{3\},\{3,4\},\{1\},\{1,3\}$, $\{1,3,4\},\{1,2,3\}\}$.

Then bi-Alex open sets are $X, \emptyset,\{1\},\{2\},\{1,2\},\{1,2,3\}$. Let $S=\{2,3\}$, then $\tau_{S}$ is $\{X, \emptyset,\{2\},\{3\},\{2,3\}\}$.

Definition 3.8. If $S$ is a subspace of bi-Alexandroff topological space $X$, we say that $H$ is open in $S$ if $H \in \tau_{S}$.

Lemma 3.9. Let $S$ is a subspace of bi-Alexandroff topological space $X$. If $H$ is open in $S$ and $S$ is bi-Alex open in $X$, then $H$ is bi-Alex open in $X$.

Proof. Since $H$ is open in $S$, then by the definition of subspace bi-Alexandroff topology, we can write $H=S \cap G$, for some set $G$ is bi-Alex open in $X$. Since $S$ is bi-Alex open in $X$ which implies that, $S$ and $G$ are both bi-Alex open sets in $X$. Thus we have $S \cap G$ is bi-Alex open set in $X$.

Lemma 3.10. Let $\left(X, A_{\tau_{1}}, A_{\tau_{2}}\right)$ be a bi-Alexandroff topological space and let $\mathscr{B}$ be base for $A_{\tau_{1}} \cap A_{\tau_{2}}$. If $\left(S, \tau_{S}\right)$ is a subspace of $\left(X, A_{\tau_{1}}, A_{\tau_{2}}\right)$, then $\mathscr{B}_{S}=\{B \cap S \quad \mid B \in \mathscr{B}\}$.

Proof. $\mathscr{B}$ is a base for $A_{\tau_{1}} \cap A_{\tau_{2}}$, and so $\mathscr{B} \subseteq A_{\tau_{1}} \cap A_{\tau_{2}}$, which implies that, $\mathscr{B} \subseteq \tau_{S}$ -(1)

Let $H \in \tau_{S}$ which implies that $H=G \cap S$, for some bi-Alex open set $G$ in $X$, and let $y \in H$ from this we get $y=G \cap S$, this means that $y \in G$ and $y \in S$. Since $\mathscr{B}$ is a base for $A_{\tau_{1}} \cap A_{\tau_{2}}$, $y \in G$ and $G \in A_{\tau_{1}} \cap A_{\tau_{2}}$, which implies that $y \in B \subseteq G$, for some subset $B$ in $\mathscr{B}$. Then $y \in B \cap S \subseteq G \cap S$. We have $H=$ 
$G \cap S$, so we can write $y \in B \cap S \subseteq G \cap S=H$. Let $\mathscr{B}_{S}=B \cap S$, then $B_{S} \in \mathscr{B}_{S}$. Thus for $H \in \tau_{S}$ and $y \in H$ there exists $B_{S} \in \mathscr{B}_{S}$ such that $y \in \mathscr{B}_{S} \subseteq H$

From (1) and (2), we have $\mathscr{B}_{S}$ is a base for $\tau_{S}$.

Definition 3.11. A function $f: X \rightarrow Y$ between bi-Alexandroff topological spaces is said to be bi-continuous if for every $b i$ Alex open set $H \subseteq Y$, the preimage $f^{-1}(H)$ is bi-Alex open in $X$.

Example 3.12. Let $X=\{a, b, c, d\}, A_{\tau_{1}}=\{X, \emptyset,\{a\},\{a, b\}$, $\{a, b, c\}\}, A_{\tau_{2}}=\{X, \emptyset,\{c\},\{c, d\},\{a\},\{a, c\}$,

$\{a, c, d\},\{a, b\},\{a, b, c\}\}$

then bi-Alex open sets are $X, \emptyset,\{a\},\{a, b\},\{a, b, c\}$ and bi-Alex closed sets are $X, \emptyset,\{b, c, d\},\{c, d\},\{d\}$.

Let $Y=\{1,2,3,4\}, A_{\tau_{1}}=\{Y, \emptyset,\{1\},\{2\},\{1,2\},\{1,2,3\}\}$, $A_{\tau_{2}}=\{Y, \emptyset,\{1\},\{3\},\{1,3\},\{2\},\{1,2\},\{2,3\},\{1,2,3\}$, $\{2,3,4\}\}$.

Then bi-Alex open sets are $Y, \emptyset,\{1\},\{2\},\{1,2\},\{1,2,3\}$ and bi-Alex closed sets are $Y, \emptyset,\{2,3,4\},\{1,3,4\},\{3,4\},\{1\}$.

Let us consider the diagrammatic representation of the function $f: X \rightarrow Y$ as follows,

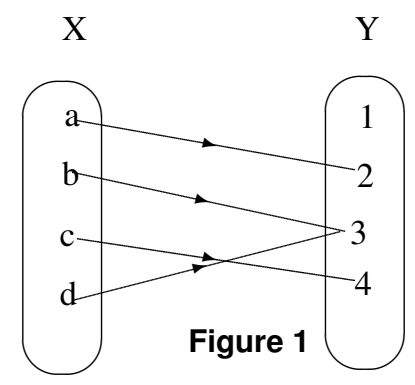

The function $f: X \rightarrow Y$ is bi-continuous, since $f^{-1}(H)$ is bi-Alex open in $X$ for every bi-Alex open set $H$ in $Y$.

Lemma 3.13. If $S$ is a subspace of a bi-Alexandroff topological space $X$, then the inclusion function $i: S \rightarrow X$ is bicontinuous.

Proof. Suppose if $G$ is a bi-Alex open in $X$, then $i^{-1}(G)=$ $G \cap S$ which is bi-Alex open in $S$ by the definiton of subspace bi-Alexandroff topology.

Lemma 3.14. If $f: X \rightarrow Y$ is a bi-continuous and if $S$ is a subspace of a bi-Alexandroff topological space $X$, then $f \mid S: S \rightarrow Y$ (restricted function) is bi-continuous.

Proof. The function $f \mid S$ equals the composite of the inclusion function $i: S \rightarrow X$ and the function $f: X \rightarrow Y$, both of which are bi-continuous function.

Lemma 3.15. If the function $f: X \rightarrow Y$ is a bi-continuous and if $T$ is a subspace of bi-Alexandroff topological space $Y$ containing the image set $f(X)$, then $g: X \rightarrow T$ obtained by restricting the range of $f$ is bi-continuous. If $T$ is a space having $Y$ as a subspace bi-Alexandroff topology, then $h: X \rightarrow$ $T$ obtained by expanding the range of $Y$ is bi-continuous.
Proof. Let the function $f: X \rightarrow Y$ be bi-continuous. If $f(X) \subset$ $T \subset Y$, we prove that the function $g: X \rightarrow T$ obtained from $f$ is bi-continuous. Let $V$ be bi-Alex open set in $T$, and $U$ be bi-Alex open set in $Y$. Then $V=T \cap U$ for some bi-Alex open set $U$ of $Y$. Since $T$ contains the entire image set $f(X)$.

$$
f^{-1}(U)=g^{-1}(V)
$$

by the elementary set theory, since $f^{-1}(U)$ is bi-Alex open set, so is $g^{-1}(V)$.

Now to prove that $h: X \rightarrow T$ is bi-continuous. If $T$ has $Y$ as a subspace bi-Alexandroff topology, note that $h$ is the composite of the map $f: X \rightarrow Y$ and the inclusion function $i: Y \rightarrow T$, it completes the proof.

\section{4. pre-bi open set and pre-bi closed set}

Definition 4.1. Let us consider $X$ be a bi-Alexandroff topological space $\left(X, A_{\tau_{1}}, A_{\tau_{2}}\right)$. A subset $S$ of $X$ is said to be a pre-bi open if $S \subseteq$ bi-int (bi-cl( $S))$ and $S$ is a pre-bi closed if the complement of $S$ is a pre-bi open.

Example 4.2. Let $X=\{a, b, c, d\}, A_{\tau_{1}}=\{X, \emptyset,\{b\},\{d\}$, $\{a, d\},\{b, d\},\{a, b, d\}\}, A_{\tau_{2}}=\{X, \emptyset,\{b\},\{d\},\{a, d\}$, $\{b, d\},\{a, b, d\},\{c\},\{b, c\},\{c, d\},\{a, c, d\},\{b, c, d\}\}$. Then bi-Alex open sets are $X, \emptyset,\{b\},\{d\},\{a, d\},\{b, d\}$, $\{a, b, d\}$ bi-Alex closed sets are $X, \emptyset,\{a, c, d\},\{a, b, c\}$, $\{b, c\},\{a, c\},\{c\}$. pre-bi open sets are $X, \emptyset,\{b\},\{d\},\{a, d\}$, $\{b, d\},\{a, b, d\},\{b, c, d\}$, pre-bi closed sets are $X, \emptyset,\{a, c, d\}$, $\{a, b, c\},\{b, c\},\{a, c\},\{c\},\{a\}$.

Remark 4.3. It is clear that, every bi-Alex open set is prebi open and every bi-Alex closed set is pre-bi closed set in general.

Theorem 4.4. Let $\left(X, A_{\tau_{1}}, A_{\tau_{2}}\right)$ be a bi-Alexandroff topological space in which each pre-bi open set a is bi-Alex open then every singleton in $X$ is either a bi-Alex open or a bi-Alex closed.

Proof. Let $a \in X$, and suppose that the singleton set $\{a\}$ is not bi-Alex open, then clearly $\{a\}$ is not pre-bi open, which implies that $\{a\} \nsubseteq b i-\operatorname{int}(b i-c l\{a\})$, so that $b i-$ $\operatorname{int}(b i-c l\{a\})=\emptyset$. We have $b i-\operatorname{int}(b i-c l\{X-\{a\}\}) \supseteq$ $b i-\operatorname{int}(b i-c l\{a\})=b i-\operatorname{int}(X-b i-\operatorname{int}(b i-c l\{a\}))=X \supseteq$ $X-\{a\}$. Thus $X-\{a\}$ is pre-bi open and hence it is bi-Alex open. Therefore $\{a\}$ is bi-Alex closed set.

Theorem 4.5. $\left(X, A_{\tau_{1}}, A_{\tau_{2}}\right)$ is a bi-Alexandroff topological space in which every subset is a pre-bi open if and only if every bi-Alex open set in $\left(X, A_{\tau_{1}}, A_{\tau_{2}}\right)$ is a bi-Alex closed set.

Proof. Let us consider $U$ be a bi-Alex open set in $X$, then we have $X-U=b i-c l(X-U)$ which is pre-bi open, which implies that $b i-c l(X-U) \subset b i-i n t(b i-c l(b i-c l(X-U)))=$ $b i-\operatorname{int}(b i-c l(X-U))=b i-\operatorname{int}(X-U)$. Thus $X-U=$ $b i-\operatorname{int}(X-U)$. Hence $X-U$ is a bi-Alex open and we get $U$ is a bi-Alex closed. 
Conversely, let $S$ be a subset of $X$, then $S-b i-c l(S)$ is biAlex open and hence bi-Alex closed. Thus we have $X-b i-$ $c l(S)=b i-c l(X-b i-c l(S))=X-b i-i n t(b i-c l(S))$. So that $S \subset b i-c l(S)=b i-\operatorname{int}(b i-c l(S))$, and hence $S$ is pre-bi open.

Theorem 4.6. Let $\left(X, A_{\tau_{1}}, A_{\tau_{2}}\right)$ be a bi-Alexandroff topological space, then the following results are equivalent.

(i) Every pre-bi open set is bi-Alex open.

(ii) Every dense set is bi-Alex open.

Proof. $(i) \Rightarrow(i i)$. Let $S$ be a dense subset of $X$. Then $b i-$ $\operatorname{int}(b i-c l(S))=X$, so that $S \subset b i-i n t(b i-c l(S))$ and hence $S$ is pre-bi open and $S$ is bi-Alex open.

$(i i) \Rightarrow(i)$. Let $A$ be a pre-bi open subset of $X$, so that $A \subset$ $b i-\operatorname{int}(b i-c l(A))=U$ (say). Then $b i-c l(A)=b i-c l(U)$, so that $b i-c l((X-U) \cup A)=b i-c l(X-U) \cup b i-c l(A)=$ $(X-U) \cup b i-\operatorname{cl}(U)=X$, and hence $(X-U) \cup A$ is dense in $X$. Thus we have $(X-U) \cup A$ is bi-Alex open. Now $A=$ $((X-U) \cup A) \cap U$, the intersection of two bi-Alex open sets, so that $A$ is bi-Alex open.

\section{5. $j$-open set on bi-Alexandroff topological space}

Definition 5.1. Let us consider $X$ be a bi-Alexandroff topological space $\left(X, A_{\tau_{1}}, A_{\tau_{2}}\right)$. A subset $S$ of $X$ is said to be $j$-bi open set if $S \subseteq b i-i n t(p r e-b i-c l(S))$ and $S$ is called $j-b i$ closed if the complement of $S$ is j-bi open.

Example 5.2. Let $X=\{1,2,3,4\}, A_{\tau_{1}}=\{X, \emptyset,\{1\},\{2\}$, $\{1,2\},\{2,3,4\}\}, A_{\tau_{2}}=\{X, \emptyset,\{1\},\{3\},\{1,3\},\{2\},\{1,2\}$, $\{2,3\},\{1,2,3\},\{2,3,4\}\}$.

Then bi-Alex open sets are $X, \emptyset,\{1\},\{2\},\{1,2\},\{2,3,4\}$. bi-Alex closed sets are, $X, \emptyset,\{2,3,4\},\{1,3,4\},\{3,4\},\{1\}$. $j$-bi open sets are, $X, \emptyset,\{1\},\{2\},\{1,2\},\{2,3\},\{2,4\},\{1,2,3\}$, $\{2,3,4\},\{2,4,1\}, j$-bi closed sets are, $X, \emptyset,\{2,3,4\},\{1,3,4\}$, $\{3,4\},\{1,4\},\{1,3\},\{4\},\{1\},\{3\}$.

Theorem 5.3. Arbitrary union of j-bi open sets is a j-bi open.

Proof. Let $\left\{S_{i} \mid i \in I\right\}$ be a collection of $j$-bi open sets in $X$, for each $i \in I$.

$$
\begin{aligned}
& S_{i} \subseteq b i-i n t\left(\text { pre }-b i-c l\left(S_{i}\right)\right) \\
& \cup_{i \in I} S_{i} \subseteq \cup_{i \in I}\left[b i-i n t\left(\text { pre }-b i-c l\left(S_{i}\right)\right)\right] \\
& =\left[b i-i n t\left(\cup_{i \in I} \text { pre }-b i-c l\left(S_{i}\right)\right)\right] \\
& =\left[b i-\operatorname{int}\left(\text { pre }-b i-c l\left(\cup_{i \in I} S_{i}\right)\right)\right] \\
& \cup_{i \in I} S_{i} \subseteq\left[b i-i n t\left(\text { pre }-b i-c l\left(\cup_{i \in I} S_{i}\right)\right)\right] .
\end{aligned}
$$

This implies that $\cup_{i \in I} S_{i}$ is $j$-bi open set.

Theorem 5.4. Arbitrary intersection of j-bi closed sets is j-bi closed.

Proof. Let $\left\{F_{i} \mid i \in I\right\}$ be a collection of $j$-bi closed sets in a space $X$, for each $i \in I$, then $b i-i n t\left(p r e-b i-c l\left(F_{i}\right)\right) \subset F_{i}$, since $F_{i}^{c}$ is an arbitrary indexed collection of $j$-bi open sets, from above theorem 5.3 we get $\cup_{i \in I} F_{i}^{c}$ is a $j$-bi open set, since $\cup_{i \in I} F_{i}^{c}=\left(\cap_{i \in I} F_{i}\right)^{c}$, which implies that $\left(\cap_{i \in I} F_{i}\right)^{c}$ is $j$-bi open set. Hence $\cap_{i \in I} F_{i}$ is $j$-bi closed set.
Remark 5.5. We will denote the j-bi interior (resp. j-bi closure) of any subset $S$ of $X$ by j-bi-int $(S)$ (resp. j-bi-cl(S)) where $j$-bi-int $(S)$ is the union of all $j$-Bi open sets contained in subset $S$ and $j$-bi-cl(S) is the intersection of all $j$-bi closed sets containing subset $S$.

Theorem 5.6. A subset $S$ of $X$ is a j-bi open if and only if $S=j-b i-\operatorname{int}(S)$.

Proof. $S$ is $j$-bi open and we know that $S \subseteq S$. Therefore $S \in\{P \quad \mid P \subseteq S, P$ is $j$-bi open $\} . S$ is in this collection and other remaining members in this collection is a subset of $S$ and clearly union of this collection is $S$. That is,

$$
\cup\{P \quad \mid P \subseteq S, P \text { is } j \text {-bi open }\}=S
$$

and hence $j-b i-\operatorname{int}(S)=S$.

Conversely, since $j-b i-\operatorname{int}(S)$ is $j$-bi open set. That is $S=j-b i-\operatorname{int}(S)$, which implies that $S$ is a $j$-bi open set.

Theorem 5.7. A subset $S$ of $X$ is a j-bi closed if and only if $S=j-b i-c l(S)$.

Proof. From the definition of $j$-bi closure, we can write $j-$ $b i-\operatorname{cl}(S)=\cap\{P \quad \mid P \supset S, \quad P$ is $j$-bi closed $\}$. If $S$ is a bi-Alex closed then $S$ is a member in $\cap\{P \mid P \supseteq S, P$ is $j$-bi closed $\}$, and each member contains $S$. Hence $S=$ $j-b i-c l(S)$.

Conversely, If $S=j-b i-\operatorname{cl}(S)$, then $S$ is bi-Alex closed, since $j$-bi-cl(S) is a j-bi closed set.

\section{6. $j$-bi-continuous function}

Definition 6.1. Let $\left(X, A_{\tau_{1}}, A_{\tau_{2}}\right),\left(Y, A_{\tau_{1}^{*}}, A_{\tau_{2}^{*}}\right)$ be bi-Alexandroff topological spaces. A function from $X$ into $Y$ is called $j$-bicontinuous if $f^{-1}(H)$ is $j$-bi open in $X$ for every $j$-bi open set $H$ in $Y$.

Example 6.2. Let $X=\{w, x, y, z\}, A_{\tau_{1}}=\{X, \emptyset,\{w, x, y\},\{w, x\}$, $\{w\}\}, A_{\tau_{2}}=\{X, \emptyset,\{w\},\{y\},\{w, y\},\{y, z\},\{w, x\},\{w, y, z\}$, $\{w, x, y\}\}$. Let $Y=\left\{v_{1}, v_{2}, v_{3}, v_{4}\right\}, A_{\tau_{1}^{*}}=\left\{Y, \emptyset,\left\{v_{1}\right\},\left\{v_{2}\right\}\right.$, $\left.\left\{v_{1}, v_{2}\right\},\left\{v_{1}, v_{2}, v_{3}\right\}\right\}, A_{\tau_{2}^{*}}=\left\{Y, \emptyset,\left\{v_{1}\right\},\left\{v_{3}\right\},\left\{v_{1}, v_{3}\right\}\right.$, $\left.\left\{v_{2}\right\},\left\{v_{1}, v_{2}\right\},\left\{v_{2}, v_{3}\right\},\left\{v_{1}, v_{2}, v_{3}\right\},\left\{v_{2}, v_{3}, v_{4}\right\}\right\}$.

Let us consider the diagrammatic representation of the function $f: X \rightarrow Y$ as follows,

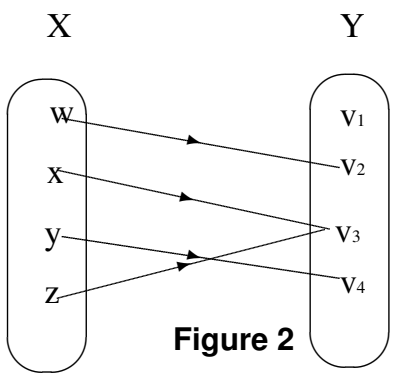

The bi-Alex open sets of $X$ are $X, \emptyset,\{w\},\{w, x\},\{w, x, y\}$ bi-Alex closed sets are $X, \emptyset,\{x, y, z\},\{y, z\},\{z\}$ 
bi-Alex open sets of $Y$ are $Y, \emptyset,\left\{v_{1}\right\},\left\{v_{2}\right\},\left\{v_{1}, v_{2}\right\},\left\{v_{1}, v_{2}, v_{3}\right\}$ bi-Alex closed sets are $Y, \emptyset,\left\{v_{2}, v_{3}, v_{4}\right\},\left\{v_{1}, v_{3}, v_{4}\right\},\left\{v_{3}, v_{4}\right\}$, $\left\{v_{1}\right\}$, and j-bi open sets in $\left(X, A_{\tau_{1}}, A_{\tau_{2}}\right)$ are, $X, \emptyset,\{w\},\{w, x\}$, $\{w, y\},\{w, z\},\{w, x, y\},\{w, y, z\},\{z, w, x\}$

j-bi open sets in $\left(Y, A_{\tau_{1}^{*}}, A_{\tau_{2}^{*}}\right)$ are, $X, \emptyset,\left\{v_{1}\right\},\left\{v_{2}\right\},\left\{v_{1}, v_{2}\right\}$,

$\left\{v_{2}, v_{3}\right\},\left\{v_{2}, v_{4}\right\},\left\{v_{1}, v_{2}, v_{3}\right\},\left\{v_{2}, v_{3}, v_{4}\right\},\left\{v_{2}, v_{4}, v_{1}\right\}$.

The function $f: X \rightarrow Y$ is $j$-bi-continuous, since $f^{-1}(H)$ is $j$-bi open in $X$ for every $j$-bi open set $H$ in $Y$.

Theorem 6.3. Let $X$ and $Y$ be bi-Alexandroff topological spaces and let the function from $X$ into $Y$. Then the following results are equivalent.

(i) $f$ is j-bi-continous.

(ii) For every subset $S$ of $X$, one has $f(\bar{S}) \subset \overline{f(S)}$.

(iii) for every $j$-bi closed set $F$ of $Y$, the set $f^{-1}(F)$ is $j$-bi closed in $X$.

(iv) For each $a \in X$ and each neighborhood $H$ of $f(a)$, there is a neighborhood $G$ of a such that $f(G) \subset H$.

Proof. We prove that $(i) \Rightarrow(i i) \Rightarrow(i i i) \Rightarrow(i)$ and then $(i) \Rightarrow$ (iv) $\Rightarrow(i)$.

$(i) \Rightarrow(i i)$, Assume that $f$ is $j$-bi-continuous. Let $S$ be a subset of $X$. We prove that if $a \in \bar{S}$, then $f(a) \in \overline{f(S)}$. Let $H$ be a neighbourhood of $f(a)$, then $f^{-1}(H)$ is a $j$ - bi open set of $X$ containing $a$, it must intersect $S$ in some point $b$. Then $H$ intersects $f(S)$ in the point $f(b)$. So that $f(a) \in \overline{f(S)}$ as desired. $(i i) \Rightarrow(i i i)$, Let $F$ be $j$-bi closed in $Y$ and let $S=f^{-1}(F)$. We have to prove that $S$ is $j$-bi closed in $X$, it is enough to prove that, $\bar{S}=S$. By elementary set theory, we have $f(S)=$ $f\left(f^{-1}(F)\right) \subset F$. Therefore if $a \in \bar{S}$, then $f(a) \in f(\bar{S}) \subset \overline{f(S)} \subset$ $\bar{F}=F$, so that $a \in f^{-1}(F)=S$, thus $\bar{S} \subset S$, so that $\bar{S}=S$ as desired.

$($ iii $) \Rightarrow(i)$, Let $H$ be a $j$-bi open set of $Y$. Set $F=Y-H$, then $f^{-1}(F)=f^{-1}(Y)-f^{-1}(H)=X-f^{-1}(H)$. Now $F$ be a $j$-bi closed set of $Y$. Then $f^{-1}(F)$ is $j$-bi closed set in $X$ from hypothesis, so that $f^{-1}(H)$ is $j$-bi open set in $X$ as desired.

$(i) \Rightarrow(i v)$, Let $a \in X$ and let it be a neighbourhood of $f a$. Then the set $G=f^{-1}(H)$ is a neighbourhood of $a$ such that $f(G) \subset H$.

$(i v) \Rightarrow(i)$, Let $H$ be a j-bi open set of $Y$ and let $a$ be a point of $f^{-1}(H)$. Then $f(a) \in H$; so that by hypothesis, there is a neighbourhood $G_{a}$ of $a$ such that $f\left(G_{a}\right) \subset H$, then $G_{a} \subset f^{-1}(H)$. It follows that $f^{-1}(H)$ can be written as the union of all $j$-bi open sets $G_{a}$, so that it is $j$-bi open set.

Remark 6.4. If the condition (iv) in above theorem 6.3 holds for the point a of $X$, we say that $f$ is $j$-bi-continuous at the point $a$.

\section{Rules for constructing $j$-bi-continuous function}

Lemma 7.1. Let us consider $X$ and $Y$ be bi-Alexandroff topological spaces and $y \in Y$. The function $c_{y}: X \rightarrow Y$ maps all of $X$ in to the single point $y$ is J-bi-continuous, where $c_{y}$ is called constant function.
Proof. Let $H$ be a $j$-bi open set in $Y$. If $y \in H$, then $c_{y}^{-1}(H)=$ $X$, which is $j$-bi open set. On the other hand, if $y \notin H$, then $c_{y}^{-1}(H)=\emptyset$, so again we get the preimage is a $j$-bi open.

Lemma 7.2. Let $X, Y$ and $Z$ be three bi-Alexandroff topological spaces. If the function $f: X \rightarrow Y$ and $g: Y \rightarrow Z$ are $j$-bi-continuous, then their composition map gof $: X \rightarrow Z$ is j-bi-continuous.

Proof. Let $H \subseteq Z$ be a $j$-bi open, then

$$
\begin{aligned}
& (g \circ f)^{-1}=\{a \in X \mid \operatorname{gof}(x) \in H\} . \\
& =\{a \in X \mid g(f(x)) \in H\} \text {. } \\
& =\left\{a \in X \mid f(x) \in g^{-1}(H)\right\} \text {. } \\
& =\left\{a \in X \mid x \in f^{-1}\left(g^{-1}\right)(H)\right\} . \\
& (g \circ f)^{-1}=f^{-1}\left(g^{-1}\right)(H) \text {. }
\end{aligned}
$$

Now, $g$ is a $j$-bi-continuous, so $g^{-1}(H)$ is $j$-bi open in $Y$ and $f$ is a $j$-bi-continuous, thus $f^{-1}\left(g^{-1}\right)(H)$ is a $j$-bi open in $X$.

\section{References}

[1] P. Alexandroff, Diskrete Raume, Recueil Mathematique, 2(44)(1937), 501-519.

[2] F. G. Arenas, Alexandroff Spaces, Acta Mathematica Universitatis Comenianae, 68(1)(1999), 17-25.

[3] I. Arockiarani, A. A. Nithya, On alexandroff space, Asian journal of current engineering and maths, 2(3)(2013), 205-207.

[4] Dhanya V. Mukundan, Introduction to quad topological spaces, International Journal of Scientific and Engineering Research, 4(7)(2013), 2483-2485.

[5] James R.Munkres, Topology (second edition), Pearson Education Limited, (2013).

[6] J. C. Kelly, Bitopological Spaces, London Mathematical Society, 3(1963), 17-89.

[7] E. H. Kronheimer, The topology of digital images, Topology and its Applications, 46(1992), 279-303.

[8] Martin M. Kovar, On 3-topological Version of $\theta$ - Regularity, International Journal of Mathematics and Mathematical Sciences, 23(6)(2000), 393-398.

[9] A.S. Mashor and M.E.EI.Monsef and S.N.EI.Deeb, On Precontinous and weakprecontinous functions, Proceedings of the Mathematical and Physical Society of Egypt, 53(1982), 47-53.

[10] Michael C. McCord, Singular homology groups and homotopy groups of finite topological spaces, Duke Mathematical Journal, 33, 465-474, 1966.

[11] D.Sasikala and I.Arockiarani, $\lambda_{\alpha}$-j-closed sets in generalized topological spaces, International Journal of Applied Science and Technology, 1(2)(2011), 200-210.

[12] D.Sasikala and I.Arockiarani, Decomposition of j-closed sets in Bi generalized topological spaces, International Journal of Mathematical Engineering and Science, 1(7)(2012), 11-18. 
[13] Timothy Speer, A Short Study of Alexandroff Spaces, New york University, (2007).

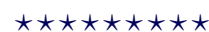

$\operatorname{ISSN}(\mathrm{P}): 2319-3786$

Malaya Journal of Matematik

ISSN(O):2321 - 5666

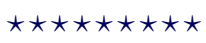

